http://dx.doi.org/10.35381/r.k.v5i4.957

\title{
Implicaciones contables de los efectos del Covid-19 en el sector camaronero del Ecuador
}

\section{Accounting implications of the effects of the Covid-19 in the shrimp sector of Ecuador}

Stalin Vladimir Paladines-Morocho stalin.paladines16@est.ucacue.edu.ec

Universidad Católica de Cuenca, Cuenca

Ecuador

https://orcid.org/0000-0003-3388-9822

Verónica Paulina Moreno-Narváez

veronica.moreno@ucacue.edu.ec

Universidad Católica de Cuenca, Cuenca

Ecuador

https://orcid.org/0000-0003-1517-6124

Lenyn Geovanny Vásconez-Acuña

lenyn.vasconez@ucacue.edu.ec

Universidad Católica de Cuenca, Cuenca

Ecuador

https://orcid.org/0000-0001-9258-3255

Recibido: 01 de junio de 2020

Revisado: 20 de junio de 2020

Aprobado: 02 de septiembre de 2020

Publicado: 24 de septiembre de 2020 


\title{
RESUMEN
}

La investigación tiene por objetivo diseñar un plan integral de revisión de los estados financieros, como mecanismo de medición de los impactos económicos y financieros generados por el Covid-19 en las camaroneras de la provincia de El Oro. Tuvo un alcance descriptivo con diseño no experimental transversal. El $86.7 \%$ de los encuestados afirmó que las empresas han diseñado estrategias para manejar de mejor forma sus necesidades de efectivo en función de sus presupuestos, ajustados a las condiciones actuales. El sector presenta altas pérdidas e incertidumbre sobre su futuro, lo cual genera problemas de financiamiento, cobertura de obligaciones, reducción de la nómina, aumento de los costos operativos, reducción de los márgenes de utilidad, la situación ha obligado a los productores a tomar decisiones sobre la marcha, para mantener las empresas en funcionamiento.

Descriptores: Administración financiera; política financiera; comercio; empresa privada. (palabras tomadas de tesauro Unesco).

\begin{abstract}
The objective of the research is to design a comprehensive plan for the review of the financial statements, as a mechanism for measuring the economic and financial impacts generated by Covid-19 in the shrimp farms of the province of El Oro. It had a descriptive scope with a non-experimental design cross. $86.7 \%$ of those surveyed said that companies have designed strategies to better manage their cash needs based on their budgets, adjusted to current conditions. The sector presents high losses and uncertainty about its future, which generates financing problems, coverage of obligations, reduction of payroll, increase in operating costs, reduction of profit margins, the situation has forced producers to make decisions on the go, to keep businesses running.
\end{abstract}

Descriptors: Financial administration; financial policy; trade; private enterprises. (words taken from Unesco thesaurus). 


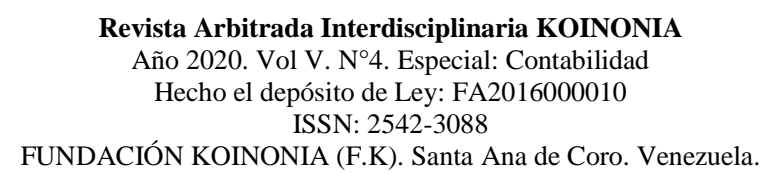

Stalin Vladimir Paladines-Morocho; Verónica Paulina Moreno-Narváez; Lenyn Geovanny Vásconez-Acuña

\section{INTRODUCCIÓN}

La preocupación mundial por los efectos de la pandemia del Covid-19, no solo es un tema de interés médico y científico, sus consecuencias y efectos se reflejan en problemas económicos y sociales. Las medidas de confinamiento para la población han reducido la oferta y la demanda de productos y servicios, afectando en gran escala el desarrollo de las actividades comerciales, industriales, agrícolas, acuícolas, entre otras.

En el caso de Ecuador el escenario no es diferente, la incertidumbre sobre los efectos de la pandemia ha afectado a los principales productos de exportación, presentándose una reducción del dinamismo en la demanda externa. Los grandes mercados como China, Estados Unidos y Europa han contraído sus economías por el aislamiento parcial o total de la población, en espera de avances médicos que permitan frenar los contagios y regresar a la normalidad (Banco Central del Ecuador, 2020).

Previo al decreto de las primeras medidas para contener los niveles de contagio, en marzo del 2020, el Ecuador esperaba un crecimiento de exportaciones no petroleras de un $3 \%$ en el caso del banano y un 7,6 \% en el caso del camarón, lo cual hacía prever un crecimiento del PIB del $0.69 \%$. La contracción de la economía a esa fecha y las proyecciones en los diferentes escenarios hacen prever una variación negativa de las exportaciones de un 5,2\%, para los sectores más representativos como el banano y camarón una caída del $13 \%$ y $21 \%$. Sumando los efectos de todos los sectores productivos, las estimaciones muestran una caída de la economía ecuatoriana para el 2021 entre un $7.3 \%$ al $9.6 \%$ (Banco Central del Ecuador, 2020).

Las implicaciones económicas generadas por la pandemia del Covid-19, deben ser cuantificadas y reflejadas en los estados financieros de las empresas, así como el detalle en sus notas, en este caso las del sector camaronero de la provincia de El Oro. Desde el punto contable las Normas Internacionales de Información Financiera (NIIF) marcan los lineamientos para registrar operaciones, y la sección 34 de la normativa para Pequeñas y Medianas Empresas (PYMES), hace relación específica a las actividades agrícolas, 


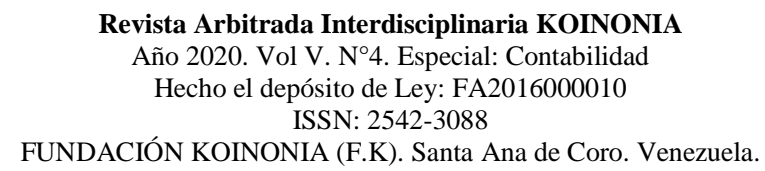

Stalin Vladimir Paladines-Morocho; Verónica Paulina Moreno-Narváez; Lenyn Geovanny Vásconez-Acuña

extracción y concesión de servicios, siendo este un instrumento para reconocer, medir y valorar la producción camaronera.

Los inversionistas, gerentes de negocios, administradores de las empresas no solo camaroneras sino de otros sectores, requieren de información financiera oportuna y razonable para tomar decisiones y así diseñar estrategias que contribuyan al cumplimiento de objetivos de mercado. La pandemia ha hecho que la mayoría de empresas reestructuren su planificación y se adapten a las nuevas condiciones, por lo tanto, es necesario conocer los efectos económicos, donde la información actualizada, permitirá desarrollar planes para el mantenimiento y crecimiento empresarial y en consecuencia de la economía del país. Por consiguiente, el problema de la investigación consiste en determinar ¿cuáles son los efectos económicos y financieros provocados por la pandemia del Covid-19 en las empresas camaroneras de la provincia de El Oro? Ante lo expuesto, la investigación tiene por objetivo diseñar un plan integral de revisión de los estados financieros, como mecanismo de medición de los impactos económicos y financieros generados por el Covid-19 en las camaroneras de la provincia de El Oro.

\section{Referencial teórico}

\section{Efectos del Covid-19 en la economía de las PYMES en expansión}

La Organización Mundial de la Salud (OMS) señala que el Covid-19, es un tipo de coronavirus que causa un sin número de problemas a la salud de las personas y afecta al sistema respiratorio. El virus tiene su origen en China, en la región de Wuhan, en donde presentó una alta tasa de contagios, que sobrepasó las fronteras hasta convertirse en una pandemia que ha afectado no solo al sistema sanitario mundial sino a su economía (Organización Mundial de la Salud, 2020).

La falta de capacidad en infraestructura hospitalaria, ha obligado a tomar medidas para minimizar el riesgo de pérdida de vidas, los gobiernos del mundo han tomado la decisión de paralizar las actividades productivas, manteniendo en cuarentena a la población, que ha provocado una caída critica de los índices económicos y una crisis global, solo 


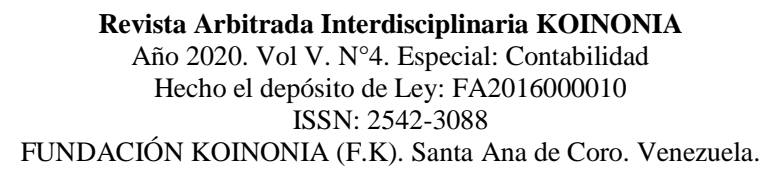

Stalin Vladimir Paladines-Morocho; Verónica Paulina Moreno-Narváez; Lenyn Geovanny Vásconez-Acuña

comparada con lo vivido en los años de la gran depresión producto de las guerras mundiales. Los impactos a nivel económico, social y de salud pública son críticos, la falta de recursos por parte de la población, la reducción de actividades productivas, pérdidas de puestos de trabajo, el cierre de fronteras y en general el debilitamiento de la oferta y demanda ha generado una reestructuración total de las políticas económicas en los países afectados.

De acuerdo al estudio del Banco de España, los escenarios aún son inciertos, los pronósticos son la base del diseño de estrategias, las cuales tienen que irse adaptando a medida que la pandemia sigue expandiéndose o conteniéndose, el no contar con referentes históricos similares o experiencias anteriores, no permite la generación de modelos adecuados que involucren a todas las variables y así lograr la reactivación económica (Banco de España, 2020). En este contexto, los impactos del Covid-19 deben ser considerados por las empresas para la elaboración de los estados financieros, es por ello, que las empresas deben ir preparando medidas para salvaguardar su capacidad de producción en la medida de lo posible, para mantenerse en funcionamiento y conocer desde ya los futuros riesgos a los cuales estarán expuestas en el futuro (PricewaterhouseCoopers Auditores, 2020).

La identificación de los efectos de la pandemia ya no solo es una actividad cualitativa, sino que es necesaria su cuantificación, en donde los responsables del área contable serán los primeros en analizar sus repercusiones y plasmarlas en los estados financieros, donde con seguridad se podrán observar cambios de la información financiera tradicional después del 31 de marzo del 2020 como gastos de cese de producción, deterioro de existencia y todos los eventos posteriores al cierre del período (Echevarría, 2020).

En el estudio de (Huilcapi-Masacón, et al., 2020), mencionan que si bien el impacto del Covid-19 es negativo, llegaría a convertirse en una oportunidad para las empresas y en especial para las PYMES, quienes han sido las más afectadas. Por consiguiente, la auto evaluación de sus prácticas productivas, de generación de ventas y de uso de la tecnología, son la base para una reestructuración de los mecanismos tradicionales que 
aplicaban antes de la pandemia, siendo el camino hacia el crecimiento y desarrollo sostenible, caso contrario sucumbirán a los efectos de la pandemia. Por lo mencionado, el contar con toda la información actualizada y con notas sobre los nuevos acontecimientos, son factores importantes para la toma de decisiones y así continuar con el cumplimiento de los objetivos de mantener las empresas en funcionamiento.

Al respecto, expertos de la (PricewaterhouseCoopers Auditores, 2020), señalan que para evaluar los impactos del Covid-19 es importante observar la información del plan contable en los siguientes referentes o variables:

1. Activos no financieros.

2. Instrumentos financieros y arrendamientos.

3. Reconocimiento de ingresos.

4. Obligaciones no financieras.

5. Aplicaciones del principio de empresa en funcionamiento.

6. Desgloses.

\section{Impacto económico-financiero en la industria camaronera a consecuencia del COVID-19}

El camarón ecuatoriano es considerado como uno de los mejores del mundo, su sabor y textura única, lo hacen muy cotizado en mercados internacionales. El posicionamiento del sector ha sido proceso de varios años donde los acuicultores han utilizado técnicas propias para el desarrollo de larvas robustecidas, empleando probióticos y con ello reduciendo el riesgo de enfermedades. Es así que, el sector de pesca y acuacultura del Ecuador tiene como producto insignia exportable el camarón, siendo el primer rubro de exportaciones no petroleras. El compromiso de las empresas productoras, aparte de lograr acreditaciones y certificaciones, es proveer un producto sustentable mediante la responsabilidad ambiental, social, seguridad alimentaria, bienestar animal y de toda su cadena de suministro (PROECUADOR, 2018). 


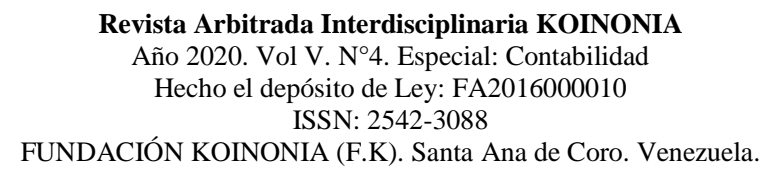

Stalin Vladimir Paladines-Morocho; Verónica Paulina Moreno-Narváez; Lenyn Geovanny Vásconez-Acuña

El Ecuador se encuentra entre los cuatro principales productores a nivel mundial de camarón junto a la India, Vietnam e Indonesia. Se ubica en el segundo lugar, y ha mostrado un crecimiento sostenible desde el año 2009 como se observa en la figura 1. Desde el año 2011 a 2019, la producción se incrementó cuatro veces, pasando de 178.000 a 635.000 toneladas exportadas (Registro Oficial № 187). En Ecuador existen alrededor de 210.000 hectáreas de camarón en cautiverio que se encuentran distribuidas y dispersas de la siguiente manera: Guayas $60 \%$, El Oro $15 \%$, Esmeraldas $9 \%$ al igual que Manabí y Santa Elena 7\% (Durán-Ganchoza, 2016).

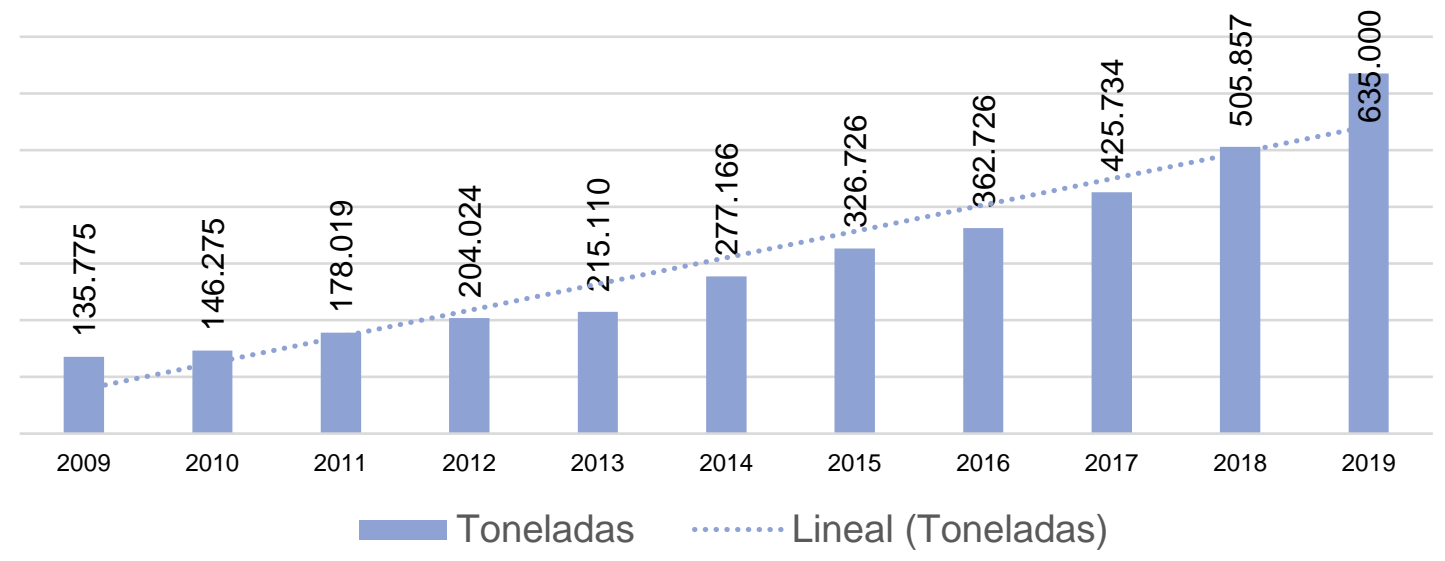

Figura 1. Producción de Camarón.

Fuente: Cámara Nacional de Acuacultura (2020).

Es importante señalar que acorde a la información proporcionada por la cámara nacional de acuacultura, el repunte del sector camaronero se debe a dos sucesos importantes bien marcados en la historia del sector. El primero debido a la duplicación de los precios que se ha presentado desde el año 2010, producto de la afectación a varios países productores de la denominada enfermedad de la mancha blanca, que no llego al Ecuador; y segundo, debido al aumento de la capacidad de producción de las empresas productoras de camarón (Jurado-Vizhñay \& Jara-Cevallos, 2016).

Los precios del camarón, fluctúan de acuerdo con los mercados internacionales, como se observa en la figura 2 el precio ha ido decreciendo. Así mismo, se evidencia que en 
los primeros meses del año 2020 a consecuencia de la pandemia mundial los precios cayeron de $\$ 2.58$ a $\$ 2.51$ USD.

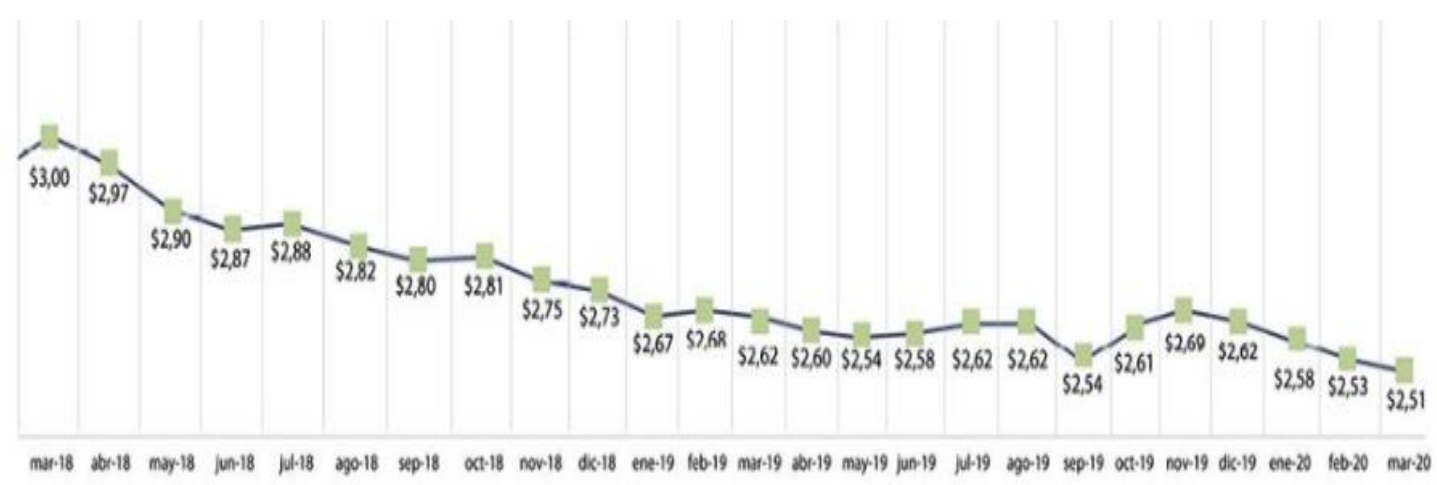

Figura 2. Evolución del precio del camarón: Promedio mensual (libras), marzo 2018 hasta marzo 2020.

Fuente: Cámara Nacional de Acuacultura (2020).

Los mercados de exportación más importante del camarón ecuatoriano son: China, Estados Unidos, España, Francia, Italia, Rusia entre otros. Con la crisis mundial, relacionada con la pandemia del Covid-19, se esperaba que en el mes de marzo de 2020, donde se detuvieron los envíos de camarón a China, se diera una caída de sus ventas en comparación a años anteriores en los mismos meses, situación que no sucedió porque el porcentaje de venta se mantuvo a niveles similares que al año anterior, vendiendo más hacia Estados Unidos y Europa (Cámara Marítima del Ecuador, 2020).

Si bien el sector camaronero ha mantenido sus niveles de producción, las nuevas restricciones sanitarias y de movilidad, perjudican no solo al sector camaronero, sino a toda la economía nacional. La caída del precio del petróleo, también presentada en marzo del 2020, el incremento del riesgo país, generan una caída en las inversiones y fuentes de financiamiento, haciendo que el país adopte medidas complejas para minimizar los graves impactos que ya afectan la debilitada economía ecuatoriana (Tene, 2020). 


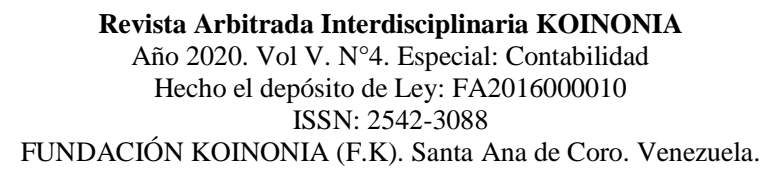

Stalin Vladimir Paladines-Morocho; Verónica Paulina Moreno-Narváez; Lenyn Geovanny Vásconez-Acuña

De acuerdo con estimaciones del Banco Central del Ecuador, el sector camaronero previo a la pandemia presentaba un crecimiento en las exportaciones del $7.6 \%$, los cuales posicionaban al camarón ecuatoriano como el principal producto de exportación. Con el cierre de las fronteras el sector descendió su nivel de exportación y producción en un $21 \%$. En este contexto, los efectos deben ser cuantificados y mostrados en los estados financieros, varias cuentas contables como los ingresos totales, gastos, depreciaciones, indemnizaciones por despidos de personal, provisiones generadas por la pandemia, entre otras, que requieran de un análisis acorde a la situación que afecta a la economía mundial.

\section{Normas Internacionales de Información Financiera (NIIF) en el Ecuador}

Las Normas Internacionales de Información Financiera (NIIF) representan un lenguaje universal para la presentación de los estados financieros, cuyo objetivo es el entendimiento de la información contable por parte de cualquier profesional en el área, empresario o inversionista. Su propósito es delinear los principios para el cálculo, identificación y formatos que muestran las actividades y movimientos económicos que inciden en las empresas a nivel mundial, siendo una herramienta fundamental para el logro de sus objetivos financieros.

La adopción de las NIIF en el Ecuador fue un proceso de convergencia, en el cual la Normativa Ecuatoriana de Contabilidad (NEC) fue sustituida por las Normas Internacionales de Contabilidad (NIC) que dieron paso a la aplicación de la NIIF en el año 2010; la normativa es de carácter obligatorio para todas las empresas que se encuentran inscritas y bajo el control de la Superintendencia de Compañías, Valores y Seguros, cuya implementación fue de carácter progresivo en función de los montos de los activos totales que poseían. Como todo proceso de cambio, este requirió de un adecuado plan, el cual, incluía un diagnóstico de la realidad del sistema contable ecuatoriano, seguido de un análisis del impacto económico y cambio social, para al final aplicar los procedimientos para la convergencia (González-Torres, et al., 2017). 


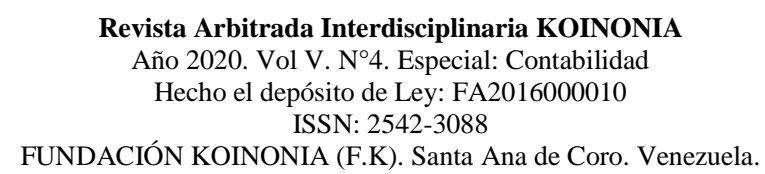

Stalin Vladimir Paladines-Morocho; Verónica Paulina Moreno-Narváez; Lenyn Geovanny Vásconez-Acuña

Antes de las NIIF las empresas en el Ecuador solo estaban obligadas a presentar el estado de situación financiera y el de resultados; la adopción de la normativa internacional generó un cambio total, se obligaba no solo a la presentación de los dos estados mencionados, sino que añadía el estado de cambios de patrimonio y el estado de flujos de efectivo, con las respectivas notas explicativas. Los cambios han contribuido a una mejora en la presentación de la información, haciéndola transparente, de fácil acceso y entendimiento para los distintos usuarios de la misma González-Torres, et al., 2017).

El objetivo esencial de los estados financieros, es servir como base para acceder a un financiamiento o realizar actividades de inversión, con el objeto de acrecentar la productividad de la entidad. El estado de situación financiera hace un compendio de toda información económica preparada y encaminada a las necesidades de información que pertenece a un grupo de usuarios, convirtiéndose en la figura económica de una entidad. Cortada a un período específico, esta detalla los resultados de todas las operaciones contables que se han realizado y son una herramienta que la junta o directivos de las empresas utiliza para la toma de decisiones (Silva-Sevilla \& García-Guzmán, 2016).

El estado de resultados o de pérdidas y ganancias es un estado único, donde se presentan todas las cuentas de ingresos, costos y gastos reconocidas en un período de tiempo. Para su elaboración es importante tomar en cuenta tres tipos de resultados integrales: a) La admisión de un estado único o dos por separado; b) Distinguir entre operaciones continuas e interrumpidas y c) presentar pérdidas y ganancia cuando la empresa posea partidas de otro resultado integral (Silva-Sevilla \& García-Guzmán, 2016).

El estado de cambios en el patrimonio, muestra las variaciones que han tenido las cuentas patrimoniales, que contienen: a) ganancias acumuladas generadas en el período. b) dividendos pagados o por pagar en el período. c) reexpresiones de lucro acumulado por designación de errores corregidos en otros períodos. d) reexpresiones de utilidades acumuladas debido a cambios de política en la empresa. El cambio del estado 


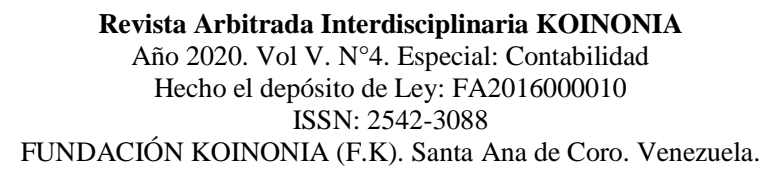

Stalin Vladimir Paladines-Morocho; Verónica Paulina Moreno-Narváez; Lenyn Geovanny Vásconez-Acuña

permite ver los contrastes de un período pasado y el período actual, convirtiéndose en una pieza fundamental para analizar el flujo de efectivo (Guamán \& Zhunaula, 2013). El estado de flujo de efectivo detalla información sobre aquellos cambios en el efectivo y sus equivalentes en un período determinado, revelando las variaciones por separado acorde a las actividades de operación, inversión y de financiamiento. Se aplican dos métodos: El directo donde se muestra los principales rubros en términos brutos, de pagos y cobros, de los procedimientos de operación de forma individual; y el indirecto que empieza cuando se presentan los resultados del ejercicio sea pérdida o ganancia (International Financial Reporting Standards Foundation (IASB), 2013).

En cuanto a la sección de las notas a los estados financieros, contienen narraciones 0 desarticulaciones sobre información financiera para una mejor explicación, brindando información detalla que no se encuentra en los registros de las cuentas para que cualquier persona comprenda el estado financiero presentado (International Financial Reporting Standards Foundation (IASB), 2013).

De acuerdo (Celi-Sánchez, et al., 2017), el proceso de adopción de las NIIF en el Ecuador tuvo como finalidad contar con elementos contables uniformes que ayuden a desarrollar adecuadas prácticas corporativas, las cuales permitan no solo contar con mecanismos de cumplimiento de los principios contables, sino añadir y utilizar elementos de supervisión y control. La aplicación de las NIIF en el país ha generado la reducción de barreras globales para los empresarios, generando mayor inversión, incremento del volumen de ventas y mayores transacciones en los mercados financieros. Aunque to mencionado no es algo genérico para todas las empresas, cada una tiene sus propias políticas con relación a sus niveles de liquidez, endeudamiento o rentabilidad.

\section{Normas internacionales de información financiera para PYMES}

Las PYMES a nivel mundial constituyen el mayor número de organizaciones productivas y generadoras de fuentes de trabajo, siendo parte de la cadena de valor de grandes corporaciones y es uno de los principales elementos de reactivación de la economía de 


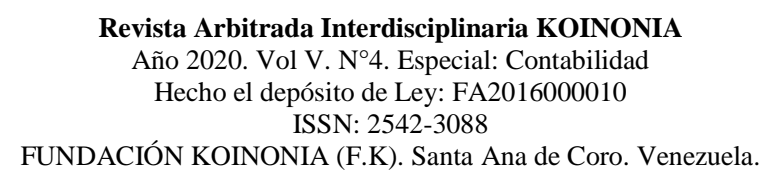

Stalin Vladimir Paladines-Morocho; Verónica Paulina Moreno-Narváez; Lenyn Geovanny Vásconez-Acuña

los países. En consecuencia, el estudio de las PYMES y su influencia, también ha abarcado a la temática contable, donde se requieren elementos adecuados para su ejecución e implementación eficaz para la presentación y uso de la información financiera (Gómez-Villegas, 2017).

Las PYMES por disposición de la norma financiera a nivel internacional, no son sujetos legales de presentar sus manejos de capital e información mercantil, por ello, solo ofrecen sus balances económicos con la única intención de informar a sus contribuyentes e inversionistas sobre su situación financiera, en otras palabras, la presentación de los estados financieros de las Pymes es opcional, no obstante, es importante para la toma de decisiones (Encalada-Encarnación, et al., 2018). En Ecuador a partir del 2012 se sugirió que las organizaciones de la categoría PYMES, presenten sus cuentas contables apegados a la normativa universal financiera, enfocándose a la presentación de los estados financieros.

Las NIIF para PYMES desde su aprobación en el año 2009, continúan en un proceso de continuos cambios y ajustes, todo en función de la realidad e importancia de este sector en la económica local y mundial. La aparición de las NIIF para PYMES responde a una necesidad de contar con adecuadas regulaciones contables para este segmento que lo integra la gran mayoría de empresas a nivel mundial. Por lo tanto, siendo las NIIF diseñadas solo para un grupo minoritario de grandes empresas, con procesos complejos y altos costos de operación contable, requieren de modificaciones para que puedan ser adoptadas y aplicadas por la mayoría de PYMES (Molina-Llopis, 2013).

Las NIIF para PYMES, tiene sus diferencias con relación a la normativa única, en general son más concretas y reducidas para cumplir con su objetivo. En general los principales cambios se resumen en los siguientes: a) no es necesaria la realización de un estado de flujos de efectivo; b) el tratamiento de todos los arriendos es operativo; c) número menor de cuentas relacionadas con las provisiones; d) no existe el reconocimiento de pago en acciones; e) no se reconocen impuestos diferidos; f) existe un modelo de costo para toda la agricultura, entre otras (Molina-Llopis, 2013). 


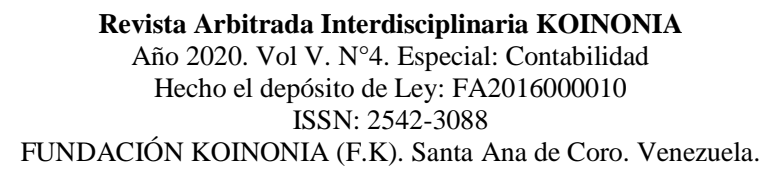

Stalin Vladimir Paladines-Morocho; Verónica Paulina Moreno-Narváez; Lenyn Geovanny Vásconez-Acuña

En el caso del presente estudio, la NIIF para PYMES en su sección 34, enfoca su trabajo y tratamiento contable a actividades especiales, las relacionadas con la agricultura, extracción y concesión de servicios. En la sección 34.2, relacionada con la política contable, indica que: a) la entidad usará el modelo del valor razonable, para sus activos biológicos, siempre y cuando este sea realizable; y b) para valorar los demás activos biológico, la empresa empleará un modelo del costo. En el caso del reconocimiento de los productos agrícolas o activos biológicos, la sección 34.3 indica que se genera cuando existe un control de los activos, producto de la ejecución de acontecimientos pasados, que produzcan beneficios económicos futuros, y puedan ser medidos de forma fiable sin costos adicionales (International Financial Reporting Standards Foundation (IASB), 2013).

Para la medición del valor razonable, en la sección 34.4 de las NIIF para PYMES indica que la entidad podrá hacer un reconocimiento de forma inicial y de ahí acorde a los informes contables, donde se cuantificarán su valor menos los costos de ventas asociados, presentados en el estado de resultados. También es necesario mencionar, que la medición deberá ser realizada en el punto de recolección y reflejará el costo a la fecha de medición con lo indica la sección 34.5 de la normativa, siempre acorde a los lineamientos de la normativa general, que en este caso es la sección 13 de las NIIF correspondiente a los inventarios (León, 2015).

Un elemento importante a tomar en cuenta en la cuantificación del valor razonable, es considerar su valor o cotización en el mercado donde esté operando. La sección 34.6 indica que se toman como referencia valores, siempre y cuando no se hayan presentado cambios significativos entre las fechas de la transacción y la fecha final del período contable. También es importante tomar en cuenta las referencias de venta de los diferentes sectores, donde los precios varían de acuerdo al producto, como kilogramos, envases, cajas, barriles, entre otros (León, 2015). 


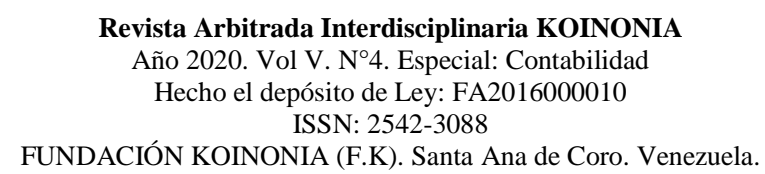

Stalin Vladimir Paladines-Morocho; Verónica Paulina Moreno-Narváez; Lenyn Geovanny Vásconez-Acuña

\section{Normativa contable aplicada en el sector camaronero}

Las actividades agrícolas son fundamentales para el desarrollo de la económica mundial. Las normativas internacionales de tratamiento contable, tienen a la NIC 41 como la encargada del registro, análisis de los procesos, cálculos y tratamientos contables de los activos biológicas (Bohušováa \& Svobodaa, 2016). La normativa como tal, analiza la transformación biológica de los activos hasta su recolección, mas no que ocurre en lo posterior, la industria camaronera encaja en la aplicación de la norma, los procesos productivos pasan por las etapas de siembra, crianza, crecimiento hasta que el producto es procesado y empaquetado para la venta (Reyes-Arana, et al., 2019).

En cuanto a la NIC 41 (Gallegos-Gallegos, 2018), menciona que es la encargada de regular los procedimientos contables que se aplican en el sector camaronero. La norma indica que un activo biológico es todo producto de especie vegetal o animal que ha atravesado una evolución biológica, originada de actividades y procedimientos empresariales relacionados con los sectores acuícola, ganadero, agrícola o similares. La normativa aporta información confiable a la cuantificación de la producción del sector camaronero, permitiendo la toma adecuada de decisiones sobre la productividad y crecimiento del sector; así como, muestra elementos que permiten minimizar los diferentes tipos de riesgo hacia los cuales está expuesto la actividad empresarial (RuizArmijos, et al., 2019)

Es importante señalar que en el Ecuador antes que se apliquen las Normas Internacionales de Información Financiera, no existía una norma local específica que permita realizar la valuación de los activos biológicos, lo cual involucraba a todos los procesos de producción hasta la comercialización. Las normativas no excluían al sector camaronero, el cual ha desarrollado criterios empíricos para reconocer, identificar y valorar la exposición y la declaración de la información financiera (Mite, et al., 2016). Cabe mencionar, que un gran número de empresas camaroneras no aplica el valor razonable en su proceso de producción tal como lo establecen las NIIF, y en general solo 


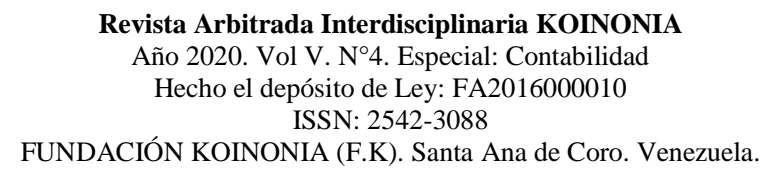

Stalin Vladimir Paladines-Morocho; Verónica Paulina Moreno-Narváez; Lenyn Geovanny Vásconez-Acuña

basan su valoración a un modelo de costos históricos para el registro y medición de sus activos biológicos. Algunas investigaciones señalan que existe una gran diferencia entre los activos estimados empleando el método de valor razonable y el de costo histórico. No obstante, la diferencia significativa de emplear un determinado método, se refleja tanto en los precios como en los estados financieros (Ruiz-Armijos, et al., 2019).

El proceso para la medición de los activos biológicos en el sector camaroneo, inicia con la adquisición de la larva, hasta su cosecha durante el período contable, acorde a las recomendaciones de la NIC 41. En la valoración se consideran factores como el tiempo de crecimiento, peso y otros costos asociados al mantenimiento en buen estado del producto y una adecuada cuantificación en cada una de las piscinas. No obstante, si bien existen diferencias de peso entre productos, sean de un mismo lote o diferente, la recomendación es la medición a un costo histórico de mercado lo cual muestra una valoración real (Ruiz-Armijos, et al., 2019).

Para calcular un adecuado costo de producción es importante conocer todas las actividades que se realizan en el proceso productivo, por tal razón se bosquejan rasgos generales que inician con la adquisición de la materia prima, que son las larvas, los diferentes tipos de balanceado para la alimentación, fertilizantes para contrarrestar enfermedades, medicamentos, bacterias, cales, y otros insumos. Con respecto a la mano de obra directa se relaciona a todo el personal necesario para la alimentación, monitoreo de crecimiento, preparación de piscinas, entre otros. Con respecto a los costos indirectos de producción, incluye los egresos de operación de equipos, suministros de mantenimiento, combustibles, impuestos, tasas y depreciaciones (Ruiz-Armijos, et al., 2019). 


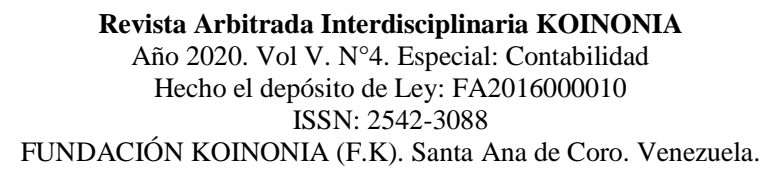

Stalin Vladimir Paladines-Morocho; Verónica Paulina Moreno-Narváez; Lenyn Geovanny Vásconez-Acuña

\section{MÉTODO}

La investigación tuvo un alcance descriptivo con diseño no experimental transversal, se realizó un análisis de los efectos que está generando la pandemia en la económica nacional y de forma específica en el sector camaronero. Así mismo, se desarrolló, revisión documental en función de la aplicación de sección 34 de la NIIF para PYMES y la NIC 41, las empresas estudiadas se encuentran vinculadas a las actividades agrícolas, pudiendo las normas ajustarse a la producción de camarón. Se aplicó el muestreo no probabilístico por conveniencia a una población de 15 empresas registradas en el Instituto Nacional de Pesca para la provincia de El Oro - Ecuador, encuestándose a 2 integrantes de cada empresa, mediante cuestionario online de varias alternativas de respuestas, el cual fue validado por juicio de experto y cálculo de coeficiente Alfa de Cronbach, teniendo un resultado de 0,81 siendo confiable para su aplicación, así mismo, los datos recopilados fueron procesados $\mathrm{n}$ función de la estadística descriptiva.

\section{RESULTADOS}

Una vez realizada la recopilación de la información a la muestra de empresas del sector camaronero, se registraron los siguientes resultados relevantes para la investigación.

\section{Impacto del Covid-19 en los ingresos y/o ganancias del sector camaronero}

Al consultar a los gerentes generales de las empresas productoras de camarón, si el Covid-19 afectó sus ingresos o ganancias, la respuesta de los encuestados fue unánime, el $100 \%$ confirmó que los precios del producto disminuyeron, y en consecuencia los ingresos y utilidades esperadas. En los primeros meses de la pandemia, el precio referencial del camarón de 14 a 16 gramos tuvo un precio promedio de $\$ 2.40$ USD, por debajo de los \$2.60 USD que se tenía en el mismo período en el año 2019. 


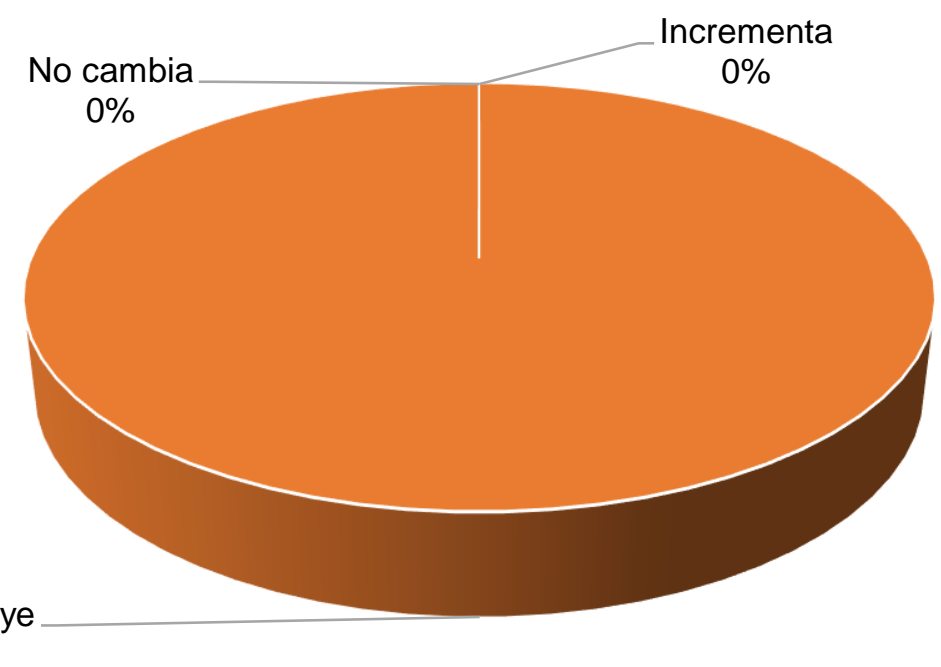

Figura 3. Resultados pregunta 1 a Gerentes Generales. Qué impacto espera obtener en los ingresos y/o ganancias del sector camaronero en este año debido al covid-19.

Las pérdidas económicas del sector camaronero durante la cuarentena por el Covid-19 son significativas, el $70 \%$ de la producción se detuvo con la pandemia. Los cambios deben ser mostrados en los estados financieros, como lo indica la sección 23 , ingresos de actividades ordinarias de las NIIF para PYMES, que hace mención a la contabilización de ingresos de actividades procedentes de la venta de bienes, la prestación de servicio, contratos de construcción, interés, regalías. Así también, en la sección 5 de la misma norma, requiere que la empresa informe su resultado integral total para un período contable, es decir, que los resultados muestren el reconocimiento del ingreso por la venta del producto.

\section{Disminución de la producción de camarón en la provincia de El Oro}

Es la primera vez que el sector camaronero del Ecuador y del mundo se ve afectado por una pandemia, en este caso la del Covid-19; si bien este virus no afecta al producto como tal, afecta a los hábitos de consumo, la oferta y la demanda se ven condicionadas por las medidas de aislamiento y movilidad impuestas para contener la propagación de los contagios, ocasionando que los principales mercados internacionales cierren sus puertas 
a la importación del crustáceo marino. En este sentido, el $66 \%$ de los gerentes consultados afirmaron que la producción ha disminuido en su totalidad, un 7\% indicó un aumento y el $27 \%$ indica que se ha mantenido en niveles similares a los generados antes de la pandemia. La Figura 4 muestra los resultados mencionados.

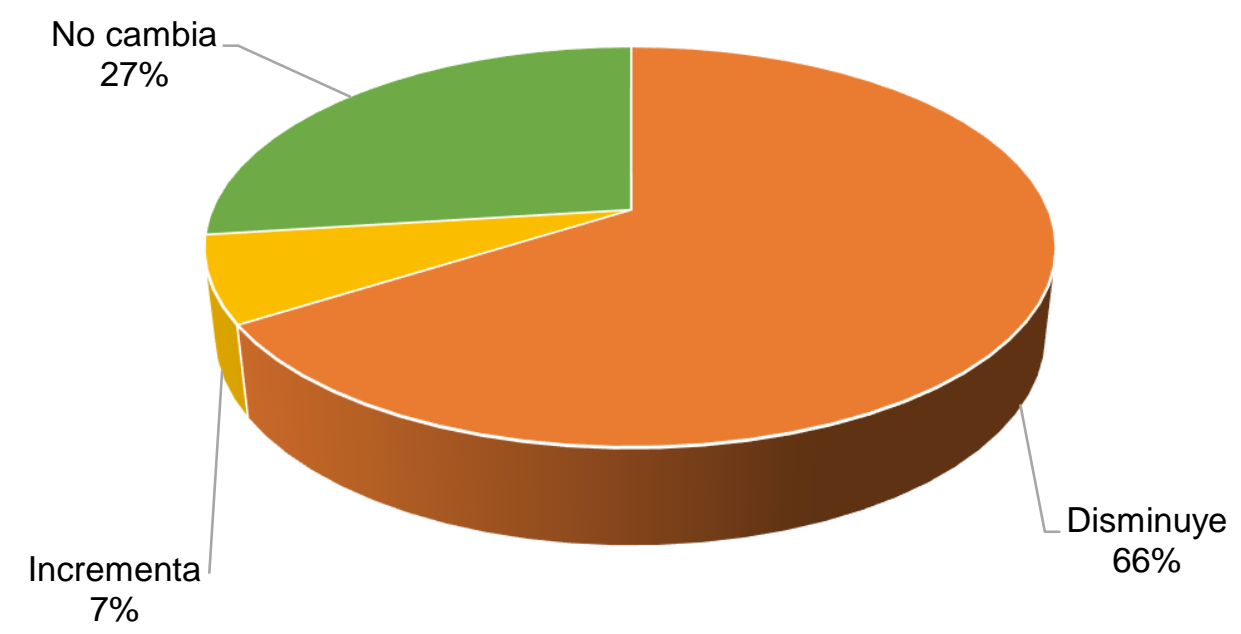

Figura 4. Resultados pregunta 5 a gerentes. Considera Usted que se disminuirá la producción del sector camaronero como consecuencia del Covid-19.

Las empresas de producción acuícola, deben contar con políticas contables que permitan registrar ajustes acordes a la situación económica presente. Los efectos, como variaciones de precio en los productos, reducción o ausencia temporal o permanente de personal que interviene en los procesos de siembra de camarón y ajustes a las asignaciones de los costos indirectos, tienen implicaciones contables que deben ser registradas, a fin de precautelar el proceso de toma de decisiones. La aplicación de la sección 10 de las NIIF para PYMES es una guía para la selección de políticas contables que se usan en la preparación de estados financieros, también abarca los cambios en las estimaciones contables y corrección de errores en estados financieros de períodos anteriores. 


\section{Liquidez y financiamiento}

Ante la recesión económica generada por el Covid-19, las respuestas de los gerentes del sector camaronero se canalizan hacia decisiones estratégicas que buscan generar oportunidades ante la adversidad. Si bien es cierto, hay que contar con los suficientes ingresos que generen liquidez para cumplir obligaciones bancarias, los resultados obtenidos indican que un $66.7 \%$ de los encuestados tienen crédito con la banca y el $33.3 \%$ dice que no posee créditos. La siguiente pregunta que hace referencia al cumplimento de pago de créditos realizados con la banca, tiene por respuesta que el $80 \%$ presentan problemas de liquidez para cancelar sus obligaciones financieras, en cambio el otro $20 \%$ indica que logra solventar sus obligaciones (ver figura 5 ).

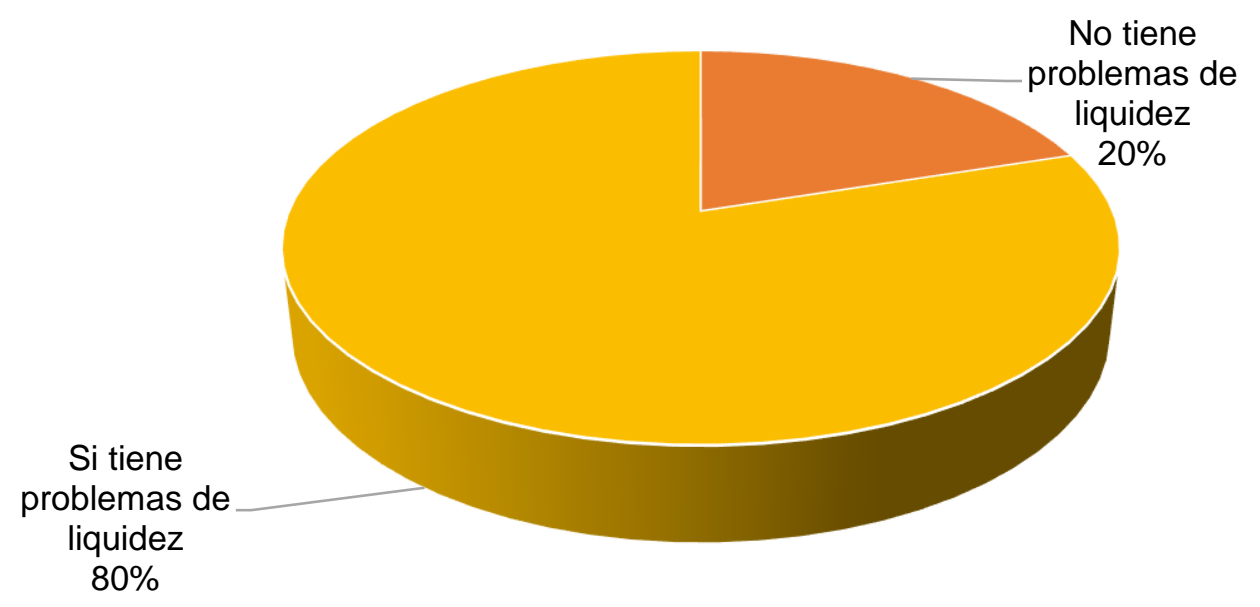

Figura 5. Resultados pregunta 14 a gerentes. El Covid-2019 afectará el cumplimiento de pago de préstamos. 
El proceso de toma de decisiones financieras frente a los efectos de la pandemia del Covid-19, se basa en los flujos de efectivo. Al respecto, el $86.7 \%$ de los encuestados afirmó que las empresas han diseñado estrategias para manejar de mejor forma sus necesidades de efectivo en función de sus presupuestos, ajustados a las condiciones actuales. La sección 7 de las NIIF para PYMES establece la información a incluir en un estado de flujos de efectivo y como presentarla, este debe proporcionar información sobre los cambios en el efectivo y equivalentes de una entidad durante el período sobre el que se informa, mostrando por separado los cambios según actividades de operación, inversión y financiación.

\section{PROPUESTA}

Una vez concluida la etapa de diagnóstico y en función de los resultados obtenidos, se concluye que el aparecimiento de la pandemia del Covid-19 ha tenido efectos en las empresas del sector camaronero nacional, la propuesta tiene como finalidad mostrar procedimientos para la revisión y evaluación integral de la información que se presenta en los estados financieros en las empresas camaroneras de la Provincia de El Oro y así medir los impactos generados en el sector. A continuación, en la figura 6 se presenta el esquema de la propuesta con los pasos a desarrollar. 


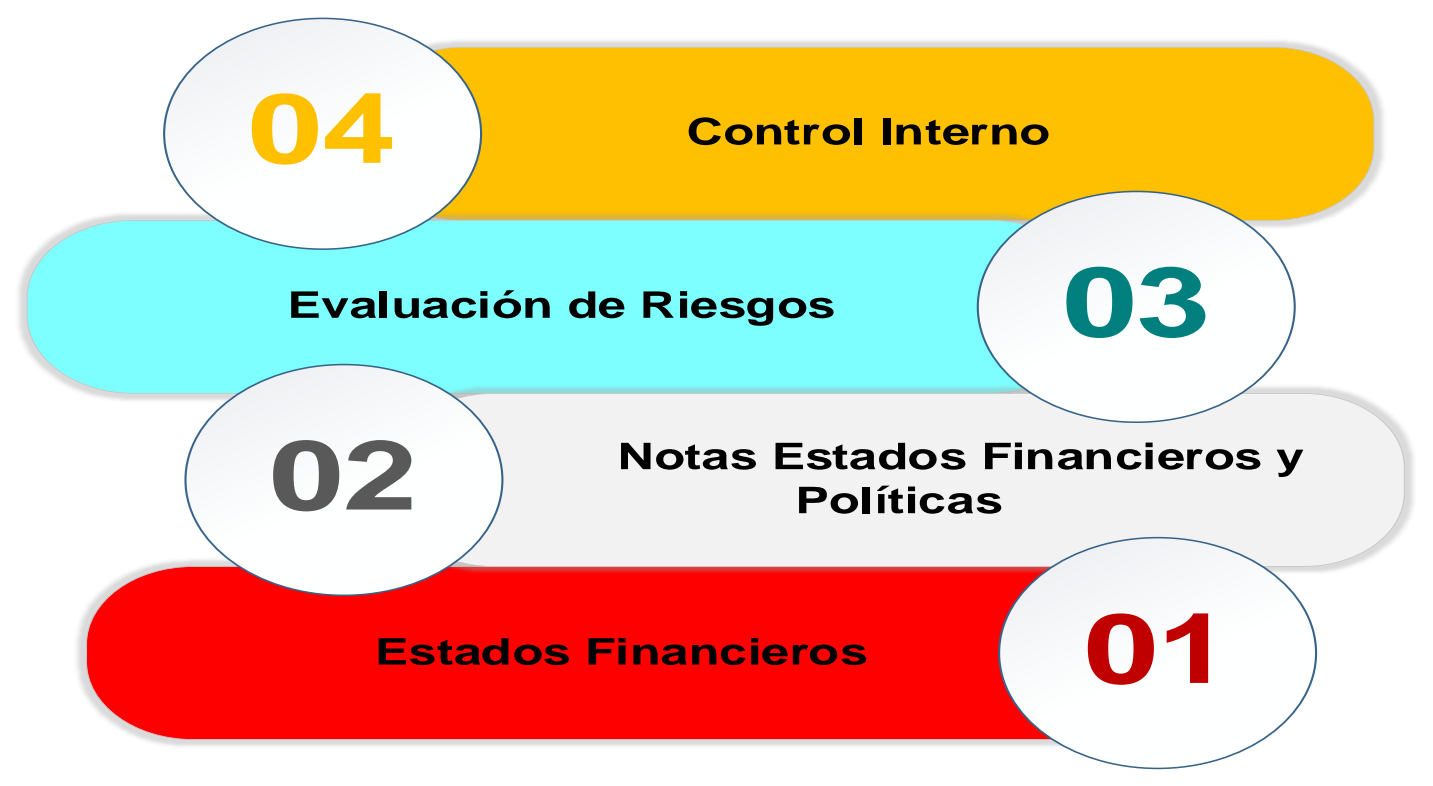

Figura 6. Plan integral de revisión financiera para medir el impacto del Covid-19.

\section{Estados Financieros}

Objetivo: proponer elementos de análisis de los efectos generados por la pandemia del Covid-19 en la preparación de los estados financieros.

Alcance: desarrollar actividades para preparar estados financieros que permitan valorar activos, pasivos, patrimonio, ingresos y egresos, revelando información real y a tiempo para salvaguardar los intereses de la empresa.

Normativa: se aplican las NIIF y las NIC.

Actividades: para la preparación de los estados financieros es necesario tomar en cuenta los siguientes pasos:

a) Analizar el deterior de los activos, acorde a la NIC 36, la cual establece los lineamientos para contabilizar los activos por un importe que no sobrepase sus valores recuperables.

b) Valorar los niveles de existencias, lo cual implica también una variación en costos fijos que son afectados por la reducción de los niveles de producción, acorde a la NIC 2. 
c) El confinamiento ha reducido el uso de las propiedades, plantas y equipos, es necesario cuantificar las amortizaciones acordes a la NIC 16.

d) Durante los primeros meses, por la falta de liquidez de toda la economía, se generaron políticas de refinanciación, en varios casos los gastos financieros fueron suspendidos, que acorde a la NIC 23 deben estar reflejados en el estado de resultados.

e) Los instrumentos financieros; préstamos, proveedores, cuentas por cobrar, arrendamientos entre otros deben revaluarse acorde a la NIIF 9.

f) Los ingresos se deben cuantificar a su valor razonable, en este caso para el sector camaronero a los precios de cotización de la unidad de mercado, acorde a los requerimientos de la NIIF 13.

g) Las acciones que ha tomado la gerencia de las empresas a causa del Covid-19, deben tomarse en cuenta como una provisión, solo en el caso que pueda cuantificarse y representen una obligación acorde a la NIC 37, como incumplimiento de contratos, indemnizaciones entre otros.

h) La reducción de la producción, genera cambios en la carga laboral, la reducción de la nómina genera obligaciones y salidas de efectivo para la empresa, se deben desarrollar acorde a la NIC 19.

i) Al reducir la producción y en consecuencia las ganancias, se deben cuantificar los impuestos sobre los beneficios, como lo indica la NIC 12, teniendo en cuenta los escenarios de incertidumbre.

j) Acorde a la NIC 34 se deben generar estados financieros intermedios, los cuales deben contener información relevante en los meses más críticos de la pandemia.

k) Con los cambios considerados se preparan los estados financieros consolidados acorde a la NIIF 10. 


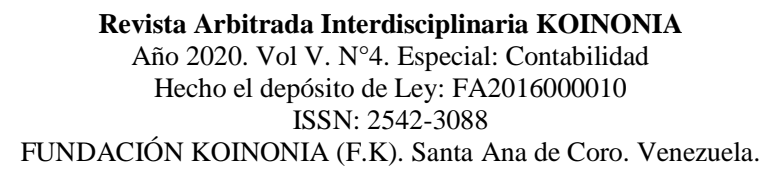

Stalin Vladimir Paladines-Morocho; Verónica Paulina Moreno-Narváez; Lenyn Geovanny Vásconez-Acuña

\section{Notas a los estados financieros y políticas contables}

Objetivo: proveer de elementos informativos para facilitar el entendimiento de los estados financieros, mostrando un detalle de los efectos en las principales cuentas por el Covid-19.

Alcance: detallar las acciones realizadas en la obtención de la información, así como proponer políticas internas para la realización de los estados financieros en función de las normativas internacionales, para evidenciar los cambios a causa del Covid-19, acorde a la NIC 10 y la sección 32 de las NIIF para PYMES.

Normativa: se aplican las NIIF y las NIC.

Actividades: la presentación de notas a los estados financieros y las principales políticas para el desarrollo de los estados financieros requieren las siguientes actividades.

a) Las notas se deberán desarrollar y mostrar información adicional tanto para el balance general, estado de resultados, estado de cambios de patrimonio y estados de flujo de efectivo.

b) Acorde a las actividades mencionadas en la fase 1, se deberán mostrar notas explicativas de los cambios generados en las distintas cuentas, indicando hechos relevantes que a simple vista no se evidencian en algún otro estado financiero.

c) Es importante mencionar que los cambios presentados son producto del impacto actual del virus o de escenarios futuros relacionados con la pandemia.

d) Las estimaciones de posibles cambios, deberán ser mencionadas y actualizadas ante los hechos presentes.

e) Es importante dar mayor relevancia en la preparación de la información y detalle de la misma a las cuentas más significativas.

f) Las notas deberán reflejar de forma específica cambios particulares a la empresa, cada entidad tiene diferentes reacciones y estrategias ante la pandemia.

g) Las fechas donde se presentan cambios considerables, son información relevante para las notas en los estados financieros. 
h) Desde el punto de vista macro es importante también incluir las acciones del gobierno tanto a nivel nacional como específicas para el sector.

i) En el caso de ser cuentas proyectadas, las notas deberán mencionar estrategias para mejorar el escenario propuesto.

\section{Evaluación de riesgos}

Objetivo: evaluar los riesgos asociados al aparecimiento de la pandemia en las empresas camaroneras de la provincia de El Oro.

Alcance: Identificar los principales riesgos y ponderarlos para su evaluación en función del impacto generado en el sector camaronero.

Normativa: normativas internas de la empresa.

Actividades: a continuación, se presentan las principales actividades para la evaluación de riesgos en las empresas a causa de Covid-19.

a) Desarrollar una matriz de riesgos, donde se incluya el nivel de impacto hacia la empresa.

b) Analizar los posibles generadores y causantes de la aparición del riesgo en las empresas.

c) Cuantificar mediante proyecciones los efectos en los estados financieros y sus respectivos indicadores a la economía de las empresas.

d) Proponer elementos de análisis comparativo para determinar si los riesgos tienen una mayor probabilidad de ocurrencia con el aparecimiento de la pandemia.

e) Analizar los impactos sociales y ambientales para el desarrollo de estratégicas de responsabilidad social. 


\section{Tabla 1.}

Tipos de riesgos asociados al Covid-19.

\begin{tabular}{ll}
\hline Riesgo & Impacto \\
\hline 1. Caída de las exportaciones & Alto \\
2. Caída de los precios & Alto \\
3. Reducción de la productividad & Alto \\
4. Caída de la rentabilidad & Medio \\
5. Aumento de precios de materiales e insumos. & Medio \\
6. Despido de personal & Medio \\
7. Incapacidad de cobertura de obligaciones & Medio \\
8. Incumplimiento de contratos de venta & Alto \\
9. Restricción sanitarias a los productos & Alto \\
10.Cierre de la empresa & Alto \\
\hline
\end{tabular}

\section{Control Interno}

Objetivo: establecer lineamientos generales, procesos y políticas contables que garanticen la veracidad de la información presentada a la junta de accionistas y organismo reguladores de control.

Alcance: desarrollar acciones que permitan contar con estados financieros ajustados y con información real, razonable y detallada en sus notas, sobre los verdaderos efectos de la pandemia en las empresas camaroneras de la provincia de El Oro.

Normativa: normas internas de la empresa y de organismos de control

Actividades: para lograr un adecuado control de la información contable generada en tiempos de Covid-19, se requiere:

a) Realizar la asignación de responsables de llevar a cabo la revisión y control de la adecuada presentación de los estados financieros. 


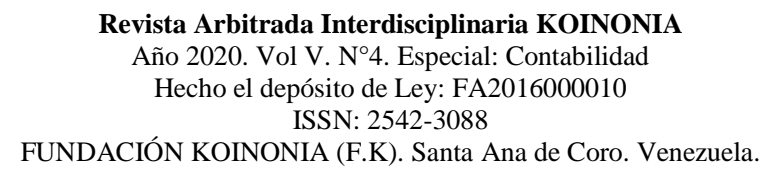

Stalin Vladimir Paladines-Morocho; Verónica Paulina Moreno-Narváez; Lenyn Geovanny Vásconez-Acuña

b) Desarrollar políticas generales ajustadas a los acontecimientos relacionados con el aparecimiento de la pandemia, que permitan evaluar los cambios en las cuentas contables y los estados financieros.

c) Analizar la información generada, contrastando las cifras obtenidas antes de la pandemia y en el momento actual, para detectar cambios y sus efectos financieros tanto en la liquidez, endeudamiento y rentabilidad.

d) La información deberá ser obtenida de medios confiables y oficiales, la empresa, organismos de control y asociaciones camaroneras proveerán de datos relevantes para el análisis de resultados.

e) Los resultados de la evaluación deben proveer de elemento de juicio para el desarrollo de estrategias para minimizar los impactos de la pandemia en el sector.

f) Documentar toda la información, la cual en un futuro permitirá evaluar si las acciones ejecutadas lograron el cumplimiento o no de su objetivo.

\section{CONCLUSIONES}

Las NIIF marcan el camino para una adecuada ejecución, presentación e interpretación de la información contable y financiera de las empresas. Las normativas desde su emisión y aplicación a nivel mundial, buscan proporcionar datos cuantitativos reales y de fácil entendimiento tanto para contadores, gerentes, accionistas, entidades de control y otros para su análisis y toma de decisiones.

Las PYMES son las principales impulsadoras de la económica local y mundial, si bien en la mayoría de casos no están obligados a llevar contabilidad, es importante contar con una normativa adaptada que ayude a optimizar sus recursos y una adecuada valoración de sus cuentas. En el caso de las PYMES del sector camaronero, la normativa internacional contable en la cual basa su trabajo es la NIC 41, direccionada al tratamiento y valoración de los activos biológicos, que en este caso se rigen a valores de mercado. El aparecimiento del Covid-19 en los primeros meses del 2020, no solo ha traído problemas de salud social, sino que ha afectado a la economía de todos los sectores 
productivos a nivel mundial y la industria del camarón no es la excepción. La demanda a nivel mundial del producto disminuyó por el cierre de las fronteras, lo cual incidió en los niveles de producción y de exportaciones, debido al temor que los alimentos se encuentren contaminados con el virus.

El sector presenta altas pérdidas e incertidumbre sobre su futuro, lo cual genera problemas de financiamiento, cobertura de obligaciones, reducción de la nómina, aumento de los costos operativos, reducción de los márgenes de utilidad, la situación ha obligado a los productores a tomar decisiones sobre la marcha, para mantener las empresas en funcionamiento.

El análisis y presentación de los estados financieros, tienen que mostrar de forma cuantitativa los eventos que han generado los cambios en las distintas cuentas a causa de Covid-19, la identificación acorde a las normativas contables, permite estudiar los factores internos y externos asociados, con lo cual se deben desarrollar medidas adecuadas para minimizar los impactos de los riesgos generados en la época actual. Tanto las entidades gubernamentales, los gremios del sector y las propias empresas deben crear políticas y estrategias para minimizar los impactos en su desempeño financiero, por tal razón es importante que el sector camaronero diseñe modelos ajustados a la nueva realidad y realizar sus cierres contables apegados a las NIIF.

\section{FINANCIAMIENTO}

No monetario.

\section{AGRADECIMIENTO}

A la Universidad Católica de Cuenca; por el apoyo en el desarrollo de la investigación. 


\section{REFERENCIAS CONSULTADAS}

Banco Central del Ecuador. (2020). El covid-19 pasa factura a la economía ecuatoriana: decrecerá entre $7,3 \%$ y $9,6 \%$ en 2020. [Covid-19 pays invoice to the ecuadorian economy: it will decrease between $7.3 \%$ and $9.6 \%$ in 2020]. Recuperado de https://n9.cl/xzql7

Banco de España. (2020). Escenarios macroeconómicos de referencia para la economía española tras el Covid-19. [Reference macroeconomic scenarios for the Spanish economy after Covid-19]. Recuperado de https://n9.cl/1y7ob

Bohušováa, H., \& Svobodaa, P. (2016). Biological Assets: In What Way should be Measured by SMEs? Procedia - Social and Behavioral Sciences 220; 62 - 69. https://doi.org/10.1016/j.sbspro.2016.05.469

Cámara Marítima del Ecuador. (2020). Coronavirus: Las ventas del camarón no se detienen, pero sí se desaceleran. [Coronavirus: Shrimp sales do not stop, but they do slow]. Recuperado de https://n9.cl/y5cem

Cámara Nacional de Acuacultura (2020). Camarón - Reporte de Exportaciones Ecuatorianas Totales. [Shrimp - Total Ecuadorian Exports Report]. Recuperado de https://n9.cl/x512

Celi-Sánchez, M., Villegas, F., \& Gaibor-Vera, F, Robles-Vizueta, F. (2017). Expectativas y realidades sobre la implementación de las NIIF en las empresas comerciales más grandes del Ecuador. [Expectations and realities about the implementation of IFRS in Ecuador's largest commercial enterprises]. Revista Espacios, 39(6); 1.

Durán-Ganchoza, F. (2016). Análisis de la Producción y Exportación de Camarón en Cautiverio Ecuatoriano..[Analysis of the Production and Export of Shrimp in Ecuadorian Captivity]. Ecuador: Universidad tecnológica empresarial de Guayaquil. Recuperado de https://n9.cl/h49a

Echevarría, J. (2020) ¿Cómo manejar los efectos financieros en las empresas? [COVID19: How to manage the financial effects on companies?]. Recuperado de https://n9.cl/qk2c5

Encalada-Encarnación, V. R., Encarnación-Merchán, O. M., \& Ruíz-Quesada, S. C. (2018). Norma internacional de información financiera: diseño e implementación en las pequeñas y medianas empresas. RIIIT. [International financial reporting standard: design and implementation in the small and medium-sized enterprises]. Revista internacional de investigación e innovación tecnológica, 6(35). 


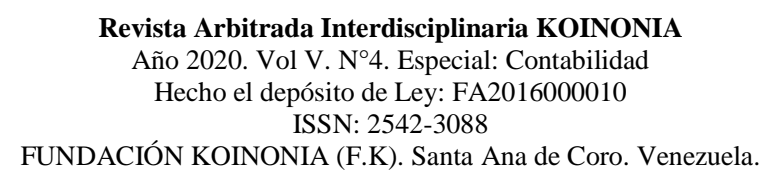

Stalin Vladimir Paladines-Morocho; Verónica Paulina Moreno-Narváez; Lenyn Geovanny Vásconez-Acuña

Gallegos-Gallegos, L. (2018). Métodos para la valoración contable de activos biológicos. [Methods for accounting valuation of biological assets]. ARJÉ. Revista de Postgrado FaCE-UC, 12(22); 342-356.

Gómez-Villegas, M. (2017). NIIF y MIPYMES: retos de la contabilidad para el contexto y la productividad. [IFRS and MSMEs: accounting challenges for context and productivity]. Cuadernos De Administración, 29(53), 49-76. https://doi.org/10.11144/Javeriana.cao29-53.nmrc

González-Torres, M, Cuenca-Jiménez; M, Higuerey-Gómez, Á, \& Villanueva-García, J (2017). Adopción NIIF en Ecuador: Análisis pre y post sobre magnitudes contables de empresas cotizadas. [Adoption of IFRS in Ecuador: Pre and post analysis on accounting figures of listed companies]. X-Pedientes Económicos, 1(1); 30-41.

Guamán, V., \& Zhunaula, M. (2013). Análisis del estado de cambios en el patrimonio según NIIf PARA PYMES. Caso práctico, cooperativa de ahorro y crédito "las lagunas", durante el período (2011-2012). [Analysis of the statement of changes in equity according to Nllf for SMEs. Practical case]. Recuperado de https://n9.cl/ogiqt

Huilcapi-Masacón, N. Úrsula, Troya-Terranova, K. T., \& Ocampo-Ulloa, W. L. (2020). Impacto del COVID-19 en la planeación estratégica de las pymes ecuatorianas [Impact of COVID-19 on the strategic planning of Ecuadorian SMEs]. RECIMUNDO, 4(3), 76-85.

International Financial Reporting Standards Foundation (2013). Una guía para micro entidades que apliquen la NIIF para las PYMES (2009). Londres: IFRS Foundation Publications Department. Recuperado de https://n9.cl/9yzi

Jurado-Vizhñay, A, \& Jara-Cevallos, G. (2016). Impacto tributario de Nic 41 en OMARSA S.A. de Durán Períodos 2012 - 2014. [Tax impact of Nic 41 on OMARSA S.A. Durán Periods 2012 - 2014]. Recuperado de https://n9.cl/st6u

León, F. (2015). Valoración de los activos biológicos de acuerdo a las NIIF PARA PYMES en las empresas bananeras. [Valuation of biological assets according to IFRS for SMEs in banana companies]. Recuperado de https://n9.cl/r2ef5

Mite, M., López, M., \& Quimí, D, Narvaez, J. (2016). Aplicación de las normas internacionales de contabilidad NIC 41 en camaroneras en Ecuador. [Application of international accounting standards IAS 41 in shrimp farms in Ecuador]. [Internet]. Revista Observatorio de la Economía Latinoamericana. Recuperado de https://n9.cl/n5qu 


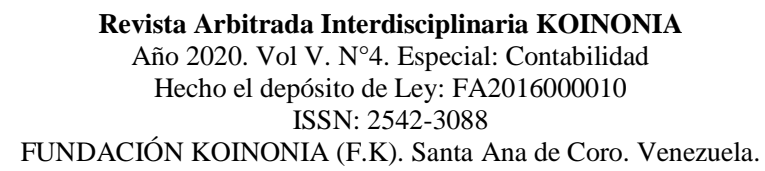

Stalin Vladimir Paladines-Morocho; Verónica Paulina Moreno-Narváez; Lenyn Geovanny Vásconez-Acuña

Molina-Llopis, R. (2013). NIIF para las PYMES: ¿La solución al problema para la aplicación de la normativa internacional?. [IFRS for SMEs: The solution to the problem for the application of international regulations?]. Contabilidad $y$ Negocios, 8(16), 21-34.

Organización Mundial de la Salud. (2020). Preguntas y respuestas sobre la enfermedad por coronavirus (COVID-19). [Questions and answers about coronavirus disease (COVID-19)]. Recuperado de https://n9.cl/gdwo

PricewaterhouseCoopers Auditores. (2020). Implicaciones contables de los efectos del coronavirus. [Accounting implications of coronavirus effects]. Recuperado de https://n9.cl/ffmi

PROECUADOR. (2018). Ficha del camarón. [Shrimp Factsheet]. Recuperado de https://n9.cl/ybiz

Registro Oficial № 187. Ley orgánica para el desarrollo de la acuicultura y pesca [Organic law for the development of aquaculture and fishing]. Recuperado de https://n9.cl/8eiup

Reyes-Arana, M. Y., Narváez-Zurita, C. I., Andrade-Amoroso, R. P., \& Erazo-Álvarez, J. C. (2019). Valoración contable de activos biológicos bajo NIIF en la empresa camaronera Biotónico S.A. Visionario Digital, 3(2.1.), 476-496.

Ruiz-Armijos, A., Narváez-Zurita, C., \& Erazo-Álvarez, J. (2019). Tratamiento del impuesto diferido a partir de la valuación de activos biológicos bajo NIIF en la industria camaronera del Ecuador. [Treatment of the deferred tax from the valuation of biological assets under IFRS in the shrimp industry of Ecuador]. Revista Arbitrada Interdisciplinaria Koinonía, 4(2), 299-320. http://dx.doi.org/10.35381/r.k.v4i2.476

Silva-Sevilla, T, \& García-Guzmán, V. (2016) Sistema Contable basado en NIIF para PYMES, en la empresa Hotel Maná del Cielo del municipio de Matagalpa, en el I semestre del año 2015.

Tene, E. (2020). Los impactos en la economía del Ecuador ocasionados por el Coronavirus [The impacts on the Ecuadorian economy caused by the coronavirus]. Recuperado de https://n9.cl/mt87q 
Revista Arbitrada Interdisciplinaria KOINONIA

Año 2020. Vol V. N${ }^{\circ}$. Especial: Contabilidad

Hecho el depósito de Ley: FA2016000010

ISSN: 2542-3088

FUNDACIÓN KOINONIA (F.K). Santa Ana de Coro. Venezuela.

Stalin Vladimir Paladines-Morocho; Verónica Paulina Moreno-Narváez; Lenyn Geovanny Vásconez-Acuña

C2020 por los autores. Este artículo es de acceso abierto y distribuido según los términos y condiciones de la licencia Creative Commons Atribución-NoComercial-Compartirlgual 4.0 Internacional (CC BY-NC-SA 4.0)

(https://creativecommons.org/licenses/by-nc-sa/4.0/ 